\title{
A GEOMETRIC-MORPHOMETRIC ANALYSIS OF THE HETEROCHELY IN THE RED SEA HERMIT CRAB "CLIBANARIUS SIGNATUS", AND ITS DEVELOPMENTAL STABILITY AND MODULARITY
}

\author{
Tarek G. Ismail \\ Zoology Department, Faculty of Science, Sohag University, Sohag, Egypt
}

\begin{tabular}{l} 
Article History: \\
Received: 25 May 2021 \\
Accepted: 10 July 2021 \\
Published Online: \\
\hline 11 July 2021 \\
Keywords: \\
Developmental stability \\
Directional asymmetry \\
Fluctuating asymmetry \\
Geometrics morphometrics \\
Modularity \\
*Correspondence: \\
Tarek G. Ismail \\
Zoology Department \\
Faculty of Science \\
Sohag University \\
Sohag, Egypt \\
E-mail: \\
t_gad_2000@science.sohag. \\
edu.eg
\end{tabular}

Article History:

Received: 25 May 202

Accepted: 10 July 2021

Published Online:

\section{Keywords:}

Geometrics morphometrics

*Correspondence:

Tarek G. Ismai

Zoology Departmen

E-mail:

edu.eg

\section{ABSTRACT}

Geometric-morphometric analysis is growingly used to quantify/analyze biological forms and discriminate among subtle variation in shape. The hermit crab "Clibanarius signatus" showed subtle heterochely; therefore, geometricmorphometric analysis helps to determine visually these shape changes. Individuals of "C. signatus" were collected from a mangrove site on the Egyptian Red Sea coast. The present study aimed to: "1" detect the different types of asymmetries in C. signatus by geometrics morphometrics, "2" inspect the responsibility of occupied shell type on the chelae asymmetry, "3" investigate the asymmetry-types' differences between sexes, "4" use the fluctuating asymmetry as an indicator for developmental stability, "5" and to investigate morphological, developmental, and functional modularity in the chela compartments. Directional and fluctuating asymmetries were detected; both showed high size levels in the males than in the females. The males revealed a high-level of shape directional symmetry and low-level of shape fluctuating asymmetry compared with the females. All investigated individuals showed larger right chela. The fluctuating asymmetry may be attributed to developmental instability as a result of occupying dextral shells. The integration and modularity of the chela compartments revealed two developmental modules, which morphologically and functionally integrated as one module. The individual variation and the fluctuating asymmetry showed similar patterns indicating that the same developmental processes are contributed to the chela shape variation. The present results are indicative signs that environmental stress is present and sufficient to produce asymmetry in chelae size/shape. However, this asymmetry was subtle, suggesting that it does not produce notable variation in the chelae functions.

\section{INTRODUCTION}

Asymmetrical morphology in chelae (heterochely) of anomuran hermit crabs is well known with a larger right chela in Paguroidae and Parapaguridae or larger left chela in the majority of Diogenidae, which also includes hermits with somewhat symmetrical chelae ${ }^{[1]}$. The morphological features of chela parts in hermit crabs are related to prospective different functions 
performed by the crab as food collection, agonistic behavior, shell investigation, and mating $^{[2]}$. The larger chela in hermit crabs has a sexual dimorphism role, besides it can be used in fighting over shells, to close off the shell aperture on threatening and/or to enhance mating success by males ${ }^{[3]}$. Therefore, all these functions lead to variations in the morphology of chelae parts and consequently shaping chelae and their handedness.

Three types of asymmetries can be observed in crabs; directional asymmetry (DA) defined as the difference between right and left sides which always biased to one side (i.e., in the same direction), fluctuating asymmetry (FA) defined as small differences between right-left sides and characterized by small random deviations from perfect symmetry (variation is normally distributed to 0 mean), while the third type is antisymmetry (AS) that is similar to DA, but the variation is non-directional (i.e., a mixture of random right- and left-sided individuals) and characterized by a bimodal and/or broadpeaked (platykurtic) distribution ${ }^{[4]}$.

Among all types of asymmetries, only FA usually employed to estimate developmental instability, which represents unstableness in phenotypic expression ${ }^{[5,6]}$ among individuals, although genotypes and the environmental factors are similar for all of them ${ }^{[7]}$. FA can produce from intrinsic stress factors such as inbreeding depression or genetic drift $^{[8]}$ or extrinsic factors such as habitat partitioning, food shortage, pollution, thermal stress, high population densities, increased intraspecific competition, and adaptation $^{[9,10]}$.

Different parts constituting an organism are organized as parts or modules, that are integrated with different degrees and relatively independent from each other ${ }^{[11]}$. This regime is referred to as modularity ${ }^{[11,12]}$. These modules are developmentally and functionally connected reflecting the morphological integration. Thus, integration and modularity are very closely related, which makes modularity is simply nested integration $^{[11]}$.
Morphological integration can be the result of developmental interactions between precursors of the traits, or it can result from environmental variance or genetic differences that affect multiple traits simultaneously. FA is considered as a tool that can be used for inferring direct interactions between the developmental pathways that produce different morphological traits ${ }^{[13,14]}$. Because FA originates from random disturbances in developmental processes, it can only produce covariation of asymmetry between two traits if the effects of the disturbances are transmitted between precursors of the traits by developmental interactions ${ }^{[12]}$. Accordingly, FA is used to investigate the developmental basis of integration ${ }^{[15,16]}$. Evidence of connection of increased FA with environmental stresses highlighted in some studies ${ }^{[6,13,17]}$ and making it accepted as a sign of developmental instability.

In the context of geometric morphometrics, hypotheses of modularity are stated in terms of the landmarks that belong to the putative modules. Under such a hypothesis, the relative positions of landmarks belonging to the same module should be integrated relatively tightly, whereas integration between modules should be weaker. By contrast, if the landmarks are partitioned into different hypothesized modules, where boundaries of these hypothesized modules do not coincide with the boundaries of true modules, the strong within-module covariation contributes to the covariation among modules, and the overall covariation among the modules of landmarks is therefore expected to be stronger $^{[12]}$. Functional modularity point to interconnections among parts to perform a given function in an organism and refers to the prospect of independent evolution of the modules and their functional integration at a given environment ${ }^{[14]}$.

Clibanarius signatus among several species of hermit crabs are found in the tidal regions of the Red Sea. Chelae of $C$. signatus populations were described as 
equal or subequal in size without any sign to their shape asymmetry ${ }^{[18]}$. The present study aimed to: "1' explore whether the geometric morphometric analyses are effective for distinguishing the different types of asymmetry in a population of hermit crab C. signatus, "2" inspect whether the occupied shell-type responsible for the environmental stress producing the chelae asymmetry, "3" investigate whether the females and the males differ in the extent of previously estimated types of asymmetries, "4" evaluate the applicability of the FA as an indicating parameter for developmental stability, "5" and to investigate the morphological and developmental integration and functional modularity in the chela compartments.

\section{MATERIAL AND METHODS Site of collection and sampling}

Individuals of $C$. signatus were handcollected in May 2018 from a single site located at about $17 \mathrm{~km}$ south of Safaga city $\left(26^{\circ} 21^{\prime} \mathrm{N}, 33^{\circ} 48^{\prime} \mathrm{E}\right)$ on the Egyptian Red Sea coast (Figure 1). The intertidal flat of the site was characterized by many mangrove trees (Avicennia marina) near the shoreline. Individuals of $C$. signatus were found scattering among mangrove trees, where they were collected and preserved in plastic vessels containing 5\% formalin. In the present work, individuals hosted in Cerithium caeruleum shells (Sowerby II, 1855) only were used. Cerithium caeruleum shells are dextral, low-spire, and have rounded aperture.

In the laboratory, hermits evacuated from their shells and sorted by sex (depending on the location of the gonopores). Specimens of both sexes were carefully examined and those with injuries or with missing or damaged or regenerated chelae were discarded from the analyses, then both right and left chelae of each specimen were dissected. The movable finger (dactyl) of each chela, was removed and the rest (propodus) was cleaned and preserved, separately, in labeled small plastic vials containing 5\% formalin.
Problems that can result from the sexual dimorphism and size-scaling of metrical characters were minimized via selecting females with shield lengths ranged from 2.41 to $4.88 \mathrm{~mm}$ and males with shield lengths ranged from 2.58 to $5.64 \mathrm{~mm}$. Therefore, 296 individuals were chosen for the following analyses, of which 192 nonovigerous females and 104 males. On the other hand, the occupied shells of Cerithium caeruleum have been measured between 2.0 and $2.7 \mathrm{~cm}$ in length.

\section{Geometric morphometric protocol for studying chelae asymmetry}

The collected specimens were divided into three datasets, the first includes the chelae of females and the second includes the chelae of males, while the third contains the data of all chelae pooled for females and males. The third dataset was used to evaluate the amount and significance of sexual dimorphism, while the first two datasets were subjected to all other analyses.

\section{Data acquisition}

The inner side of both the right and left propodus for the two sexes of $C$. signatus was photographed using a Canon digital camera (power shot A590) attached to a binocular zoom stereo microscope. Sixteen anatomical landmarks were digitized on homologous structures for both propodi (Figure 2) using tpsDig 2.22 software $^{[19]}$. These landmarks were used to cover the whole propodus structure providing a more realistic visualization of the shape features ${ }^{[20]}$. Inspecting measurement error was performed on all specimens through photographing each specimen once, and each image was digitized twice. Previous step is important to guarantee the FA can be detected and not affected by $\mathrm{DA}^{[6]}$. Imaging and digitizing were done by the author on different sessions. In the present work, the propodus has been referred to as chela.

\section{Size analysis}

For each individual, the size of both chelae was measured as a centroid size (the square root of the total squared distances between 
each landmark and the configuration centroid). The centroid size (CS) measures the overall size and its significance emerged from its mathematical independence from the shape ${ }^{[21]}$. CS was log-transformed to avoid any deviation from linearity and to scale all values in the comparisons ${ }^{[21]}$. The
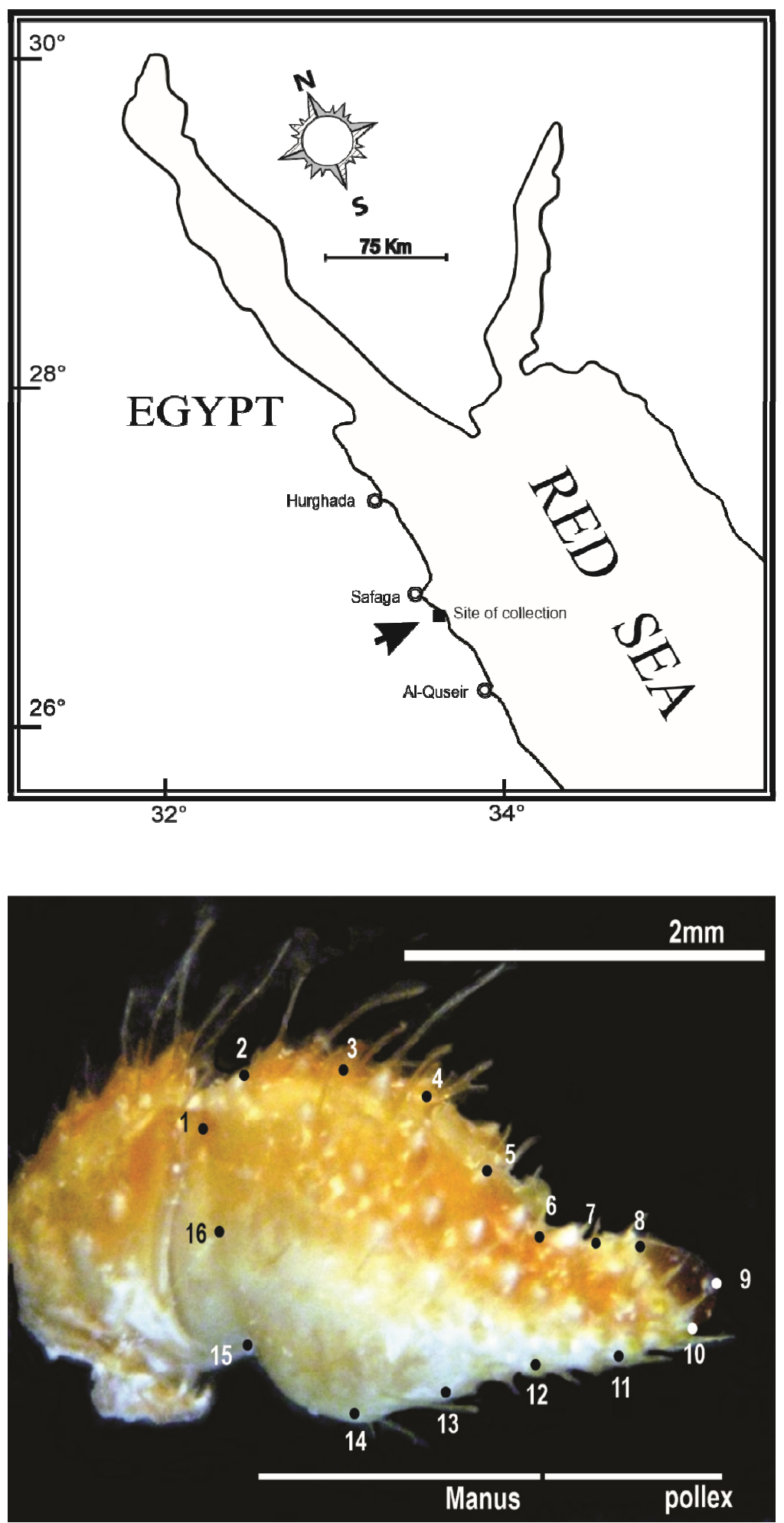

one-way ANOVA test was performed to investigate the significance of $\log \mathrm{CS}$ variations between the right and left chelae of each sex, as well as between the corresponding chelae of the two sexes (size sexual dimorphism).
Figure 1: Map for the Egyptian coastline of the Red Sea showing the location of collecting site (arrow), located at about $17 \mathrm{~km}$ south of Safaga city $\left(26^{\circ} 21^{\prime} \mathrm{N}\right.$, $\left.33^{\circ} 48^{\prime} \mathrm{E}\right)$.
Figure 2: Diagrammatic drawing of the inner side of the right chela of $C$. signatus showing the location of 16 morphological landmarks used in the geometric morphometric analysis of matching asymmetry. 


\section{Shape analysis}

Shape asymmetry of right-left chelae was investigated through the generalized Procrustes analysis (GPA) for landmark configurations with matching symmetry ${ }^{[22,23]}$ using SAGE (version 1.04) and MorphoJ (version 1.07) software ${ }^{[24,25]}$. First, configurations of left chela were reflected to their mirror images by multiplying their landmark $\mathrm{x}$-coordinates by $(-1)$ to match their right counterparts. Then, all configurations were translated to the same centroid, scaled geometrically to unit size (i.e., coordinates 0,0 ) and finally, each configuration was rotated to minimize the squared distances between homologous landmarks of configurations and their average shape. Asymmetry in right-left chelae for shape and size variations was quantified through Procrustes ANOVA and two-factor ANOVA for the three datasets separately, using superimposed configurations as dependent variables, individuals as a random effect, and sides (right and left chelae) as a fixed effect ${ }^{[26]}$. For asymmetry of shape sexual dimorphism, sex was entered as an additional fixed effect. By this, the shape and size variations were partitioned into symmetric (among individuals) and asymmetric (within individuals) components. Random effect stands for individual shape variations (symmetric variations), sides represent directional asymmetry (DA), the interaction between the individuals, and sides corresponded to fluctuating asymmetry (FA) among individuals, while measurement error stands for digitization. The significance of "individuals $\times$ sides" indicates that the morphological variation is caused by the interaction between the individuals' variation and the variation between each of the right and left chelae, reflecting the presence of FA. Procrustes ANOVA assumes that the variation is isotropic (i.e., equal distribution of configurations in all directions of the variation around the average landmark configuration), which was considered inaccurate when studying biological structures ${ }^{[27]}$. Therefore, performing permutation tests ${ }^{[28]}$, which avoid the presumption of isotropic variation, were necessary to inspect the presence of FA and DA. After this step, the replicate configurations for each of the right and left chela were averaged and used for subsequent analyses.

The presence of antisymmetry (AS), in females' and males' chelae, was investigated by calculating kurtosis $(\mathrm{K})$ for shape data of right-left distribution ${ }^{[6]}$ as well as, by the scatter plots of the differences between corresponding landmarks of the right and left sides. The clustering of the points as a bimodal distribution in the scatter plots refers to the presence of $\mathrm{AS}^{[6]}$.

An exploratory test for allometry (the effect of size on shape variation) was executed by regressing both individual means of shape symmetrical components on individual means of $\log \mathrm{CS}$ and shape asymmetric components on the asymmetry of $\log$ CS. The independence of shape from size was tested using the permutation test with 10000 runs $^{[25,28]}$. Results showed the presence of a very small allometric effect for both symmetric $(2.7 \%, P<0.0001)$ and FA components $(2.4 \%, \quad P<0.0001)$ of total shape variations for females. While, males revealed no allometric effect for both the symmetric and FA shape variations $(P>0.05)$ and regression model explained very low values $(1.4 \%$ and $1.7 \%$ of the total variance, respectively). Although, there was no need for size correction, the subsequent analyses performed on the residuals from multivariate regression to ensure eliminating the effects of size ${ }^{[29]}$ because the allometry can lead to comprehensive integration over the whole morphological structure and counteract modularity ${ }^{[12]}$.

Principal component analysis (PCA) was used to explore the patterns of shape changes in the individual (symmetric) and asymmetric (DA and FA) elements in the datasets of females and males separately, relying on the covariance matrices ${ }^{[22]}$. The analysis produces new shape variables (PC scores) for each dataset, which were used to perform a multivariate analysis of 
variance (MANOVA) using the LawleyHotelling trace test $\left(\mathrm{T}^{2}\right)$ to determine whether there were individual variations, and DA and FA differences in right-left chelae shapes among individuals of each sex. The PC scores (obtained by SEAG software) were determined for individual variations, DA and FA, and those explained more than $90 \%$ of the total variance were used as dependent variables, while sides as the independent variable. The Procrustes distance was used as a measure for shape differences between corresponding landmarks of two chelae mean shapes ${ }^{[30]}$. Besides, the similarity of DA vectors between females and males was estimated using vector correlations (VC) relative to landmark displacements regarding their directions and relative magnitudes ${ }^{[31]}$, while the significance of $\mathrm{VC}$ was evaluated with a Monte-Carlo test with 10,000 iterations.

The hypothesis of similarity between the corresponding PCs for sources of variation (i.e., individual variation and FA) was tested by comparing angles $(\alpha)$ between these respective PCs for each sex separately ${ }^{[32]}$. Also, the previous hypothesis was tested by comparing the corresponding PCs for each source of variation between sexes. Developmental stability (DS) level was calculated for both sexes using FA10 $\alpha$ index, which represents the level of shape $\mathrm{FA}^{[6]}$, and the significance of differences between sexes was estimated via Levene's test ${ }^{[33]}$.

\section{Integration and modularity}

The pattern of morphological integration in the chela of both sexes was investigated for individual variations and FA of shape, using Partial least squares (PLS) analysis depending on the covariance matrices of the individual variations and asymmetric component ${ }^{[34]}$. PLS was performed between two sets of landmarks (representing manus and pollex) within the same configuration with 10000 rounds permutation test. The landmarks of the two sets obtained by placing a boundary between manus (LM; 1 , $2,3,4,5,13,14,15,16)$ and pollex (LM; 6,
$7,8,9,10,11,12)$ parts. The analysis extracts pairs of PLS axes, one for each set, pointed out the degree of contribution of each set to the total covariation and quantified the independent features of shape covariation ${ }^{[12,16]}$. The overall integration between manus and pollex was measured by $R V$ coefficient and PLS correlation ( $\mathrm{r}_{\mathrm{PLS}}$ ) and the null hypothesis was the independence of variation between the manus and pollex ${ }^{[35]}$. The covariation between the two parts is considered weak if $R V<0.5^{[36]}$. Then, the similarity between vectors of PCA and PLS analyses, related to individual variations and FA, was conducted using angular comparisons of previous vectors for females and males, because these angles are a direct and axiomatic estimate for the resemblance of two vectors in shape tangent space. Angles are ranged between $0^{\circ}$ and $90^{\circ[25]}$. The significance of the previous analyses examined with a permutation test with 10000 runs $^{[28]}$. Developmental integration (canalization level) was explored by testing the hypothesis that the same developmental processes (developmental integration) generated the shape of individual variation and shape of FA, via comparing covariance matrices of both individual variation and "individual $\times \mathrm{FA}$ " for each sex through computing covariance matrices correlation with diagonal entries of the matrices included and excluded ${ }^{[22]}$. The significance of correlation against the null hypothesis of complete dissimilarity assessed through the matrix permutation test (with 10000 runs). This test distinguishes between direct developmental interaction pathways and parallel developmental pathways.

Also, the previous hypothesis was tested using another two ways; the first by comparing the values of angles obtained from eigenvectors pairs of covariance matrices with the expected angles between random vectors pairs ${ }^{[37]}$, since these angles reflected the similarity too. The values of $(P)$ were computed against the null hypothesis of the vectors dissimilarity ${ }^{[38]}$ and corrected with Bonferroni correction. 
The second by using the among-individual variance via computing Procrustes distances between R-L chelae for both sexes. Significance of differences in FA shape between sexes was estimated by Levene'stest ${ }^{[39]}$.

Functional integration was tested using the modularity hypothesis that the chela is partitioned functionally into a wide manus module and a wedged fixed finger or pollex module, although no clear boundary delineates the pollex from the manus (Figure 2). This assumption depends on form and function links and investigates whether the manus and pollex are integrated functionally or partitioned into different modules $^{[12]}$. The landmarks of chela were divided into two neighboring subsets within the whole configuration (the manus, LM: 1, 2, 3, 4, 5, 13, 14, 15, 16 and pollex, LM: 6, 7, 8, 9, 10, 11, 12) and their covariance were examined.

The strength of integration was measured with the Escoufier's $R V$ coefficient (implanted in MorphoJ software) and its significance tested with 10000 permutation rounds, where low values of $R V$ reflect it's near to the lowest part of the distribution and hence the hypothesis is accepted. In the present work, the considered total number of alternative partitions of the configuration of two spatially contiguous subsets containing the same number of landmarks as the hypothesized modules was 11440 calculated according to the formula of Klingenberg ${ }^{[12]}$, which provide a plausible description of the distribution of the $R V$ coefficient. The calculated $R V$ was compared with $R V$ from the alternative partitions and when it falls in the lower tail of observed $R V S$ distribution for alternative partitions or has the lowest value, the hypothesis of modularity is accepted. Significance was computed as the proportion of 11440 random partitions showing lower $R V$.

\section{RESULTS}

\section{Measurement errors}

The results of two-factor ANOVA for CS and Procrustes ANOVA for shape showed similar results, where digitizing measurement errors for chelae of females and males led to very small and negligible size and shape variations compared with individual variations (Table 1). Also, comparing mean square (MS) values revealed that the interaction

Table 1: Two-way ANOVA and Procrustes ANOVA analyses for size and shape of right-left chelae asymmetry for the whole specimens of $C$. signatus. The analyses assess the measurement errors.

\begin{tabular}{|c|c|c|c|c|c|c|}
\hline & \multicolumn{3}{|l|}{ Females } & \multicolumn{3}{|l|}{ Males } \\
\hline & $\%$ Exp Var & MS & df & $\%$ Exp Var & MS & df \\
\hline \multicolumn{7}{|c|}{ Two-Way ANOVA - Centroid Size } \\
\hline Individuals & $77.94 \%$ & $10608.66^{* \bullet}$ & 191 & $56.97 \%$ & $8929.09^{* \bullet}$ & 103 \\
\hline Sides & $11.10 \%$ & $288879.83^{* \bullet}$ & 1 & $25.44 \%$ & $410658.29^{* \bullet}$ & 1 \\
\hline Individuals $\times$ sides & $10.94 \%$ & $1489.55^{\star \bullet}$ & 191 & $17.59 \%$ & $2756.73^{* \bullet}$ & 103 \\
\hline Measurement errors & $0.011 \%$ & 0.778 & 384 & $0.005 \%$ & 0.382 & 208 \\
\hline \multicolumn{7}{|c|}{ Procrustes ANOVA - Shape $\left(\mathrm{MS} \times 10^{3}\right)$} \\
\hline Individuals & $43.26 \%$ & $0.300^{* \bullet}$ & 5348 & $42.69 \%$ & $0.348^{* \bullet}$ & 2884 \\
\hline Sides & $22.62 \%$ & $30.010^{*^{\bullet}}$ & 28 & $26.92 \%$ & $22.616^{*^{\bullet}}$ & 28 \\
\hline Individuals $\times$ sides & $33.72 \%$ & $0.234^{* \bullet}$ & 5348 & $30.18 \%$ & $0.246^{* \bullet}$ & 2884 \\
\hline Measurement errors & $0.41 \%$ & 0.0013 & 10752 & $0.20 \%$ & 0.0008 & 5824 \\
\hline
\end{tabular}

*: $P$ (parametric) $<0.0001 ; \bullet: P$ (permutation) $<0.001$; MS: mean squares; \% Exp Var: \% explained variance; df: degrees of freedom. 
(individuals $\times$ sides), which refers to FA, was highly significant and its value is largely exceeded than that of the measurement errors. This suggests that measurement errors are not of importance for the following analyses of FA in the datasets, and the results of both size and shape are valid.

\section{Asymmetry in chela size}

Analysis of variance using $\log$ CS showed that the right chelae of females and males were significantly larger than the left ones (Females: $\mathrm{F}=49.32, P<0.001$; Males: $F=59.03, P<0.001$ ) (Figure 3). This reflects chelae-size variations among individuals in both sexes. On the other hand, the two-factor ANOVA for log CS of females and males revealed the presence of both directional (sides) and fluctuating (individuals $\times$ sides) asymmetries in right-left chelae size (Table 1).

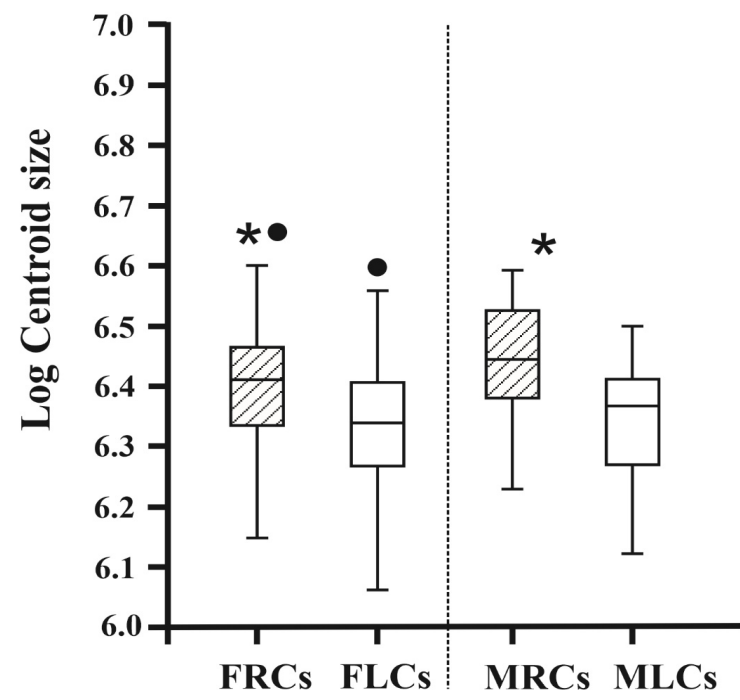

Figure 3: Size variations for the right and left chelae of the females and males of $C$. signatus. The size was measured using $\log$ centroid size. The significance of size variation was investigated by using one-way ANOVA. FRCs: Female right chela centroid size; FLCs: Female left chela centroid size; MRCs: Male right chela centroid size; MLCs: Male left chela centroid size. *: $P$ (parametric) $<0.0001{ }^{\bullet}: P$ (permutation) $<0.001$.

\section{Asymmetry in chela shape}

Females and males shape data showed significant positive kurtosis (i.e., leptokurtic distribution) ( $\mathrm{K}$ values; Females $=8.26$, Males $=5.65 ; P<0.05)$. Hence, absence of platykurtic reflects absence of antisymmetry in both males and females. This result was supported by scatter plots of individual right-left differences for both sexes, where points did not form a bimodal distribution (not shown).

The Procrustes ANOVA analysis revealed that in both females and males, individuals' variations accounted for the largest portion. Compared with directional asymmetry, the fluctuating asymmetry was contributed by a large percentage of the total variation in both females and males (Table 1). Furthermore, sex affected the observed variations (Procrustes ANOVA for 3rd dataset; sex: $\mathrm{F}=26.5$, Pillai trace $=0.63$, $P<0.0001)$. For females and males, Hotelling trace test $\left(\mathrm{T}^{2}\right)$ confirmed results of Procrustes ANOVA regarding existence of both types of asymmetries (Table 2).

\section{Patterns of shape variation}

PCA for pattern of R-L chela DA showed $100 \%$ variation in both females and males (Figure 4). The differences between right and left chelae average shapes were highly significant, but subtle, for both sexes (females: Procrustes distance $=0.06$; males: Procrustes distance $=0.07$; both $P<0.0001)$. The directional asymmetry variations were corresponding to the lowering and expansion of manus, as well as, a slight narrowing of pollex for right chela. Corresponding landmarks displacements of R-L chelae between two sexes showed some similarity (Figure 4), however, comparing DA vectors revealed that displacements of landmarks are significantly different (females versus males; Procrustes distance $=0.039, P<0.0001)$. The previous similarity of landmarks displacements was quantified using correlations between DA vectors of sexes, which was significant and positive $(\mathrm{r}=0.83 ; P<0.001)$. 
Table 2: MANOVA test for directional and fluctuating asymmetries in right-left chelae of $C$. signatus.Lawley-Hotelling trace $\left(\mathrm{T}^{2}\right)$ was used as a statistical test.

\begin{tabular}{|c|c|c|c|c|c|c|c|c|}
\hline & \multicolumn{4}{|l|}{ Females } & \multicolumn{4}{|l|}{ Females } \\
\hline & $\mathrm{T}^{2}$ & df1 & $\mathrm{df} 2$ & $\mathrm{~F}$ & $\mathrm{~T}^{2}$ & $\mathrm{df} 1$ & $\mathrm{df} 2$ & $\mathrm{~F}$ \\
\hline Individuals & 235473.7 & 149744 & 147335.5 & $1.56^{\star \bullet}$ & 162439.4 & 80752 & 78361.5 & $1.99^{\star \bullet}$ \\
\hline Sides & 48998.1 & 784 & 73146.7 & $62.2^{*^{\bullet}}$ & 48998.1 & 784 & 73146.7 & $62.2^{*^{\bullet}}$ \\
\hline Individuals $\times$ size & 79040757.6 & 149744 & 148115.2 & $524.9^{* \bullet}$ & 39916903.5 & 80752 & 79137.7 & $489.4^{\star \bullet}$ \\
\hline
\end{tabular}

*: $P$ (parametric) $<0.0001 ; \bullet: P$ (permutation) $<0.001$; df1: degrees of freedom between groups; df2: degrees of freedom within groups; F: F test statistics.
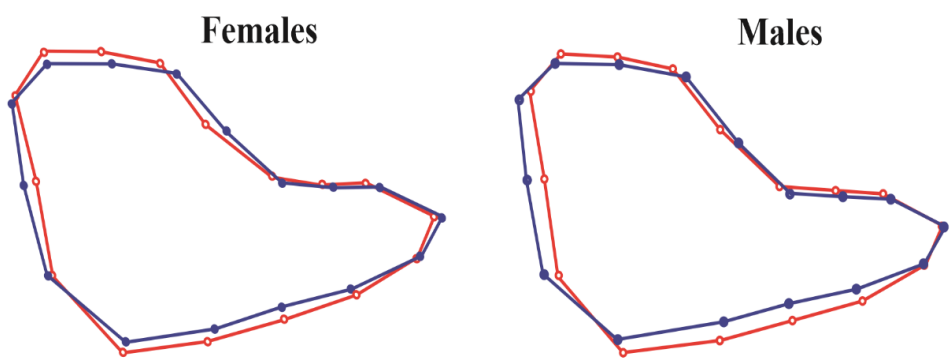

Figure 4: Directional asymmetry in the females and males of $C$. signatus. Wire-frame diagram showing the shape variations between configurations of the right (blue circles and line) and reflected left (red circles and line) chelae. The analysis depends on the residuals from the regression of shape on log-transformed centroid size.

The patterns of individual variation and fluctuating asymmetry were visualized through PCA and showed regular spread across PC scores for females and males. First three PCs of individual shape variation accounted for $44.4 \%$ and $59.0 \%$ of the individual variations for females and males, respectively (Figure 5a,b). While those of fluctuated asymmetries accounted for $43.1 \%$ and $47.6 \%$ for females and males, respectively. Mostly, the first seven PCs for both sexes accounted for more than $70 \%$ of variation regarding individual variations and FA, taken into consideration that most of variation among individuals was a little more than for FA.

Generally, the patterns of variation displayed by PCA reflected distinct series of landmark displacements. For individual variation, PC1 reflected a similar pattern of variation between the chelae of both sexes and mostly influenced the shape of the manus resulting in their heighten and narrowing. Since PCs directions is arbitrary, therefore their movement can be reflected by $180^{\circ}$, which mean that $\mathrm{PC} 1$ can be described as variation between heighten and narrowing or shortening and widening. The PC2 primarily associated with shortening (or elongation) of pollex with a blunt tip in females (the opposite in males). Also, the PC3 displayed reversed shape variation between two sexes. The manus is narrow and high, while pollex is slightly wide and blunt in females. In males, the manus is somewhat wide, while pollex is narrow.

PCA for FA patterns of variation showed little similarity to those for individual variation (Figure 5a,b). PC1 revealed that most of the variations located in the manus region, which was higher and narrow in females (opposite in males). PC2 disclosed a slight forward growing for pollex tip in the two sexes, while variations on PC3 presented an increase in the width of manus and pollex in both sexes. 
For each sex, comparing the first three PCs for individual variation and their corresponding PCs for FA suggested that the patterns of shape variation for females were similar between corresponding three PCs (PC1s: $\alpha=35.6^{\circ}$, PC2s: $\alpha=43.6^{\circ}$, PC3s: $\left.\alpha=31.3^{\circ} ; P<0.0001\right)$. The results referred that the similarities between the PC3s of fluctuating asymmetry and individual variation were closer than those of the PC1s and PC2s. As for males, there was a resemblance between corresponding
PC2s and PC3s (PC2s: $\alpha=40.6^{\circ}, P<0.001$; PC3s: $\left.\alpha=62.5^{\circ} ; P<0.01\right)$, but not PC1s $\left(\alpha=78.5^{\circ}, P=0.293\right)$. On the other hand, the shape features for individual variation between sexes was similar (PC1s: $\alpha=42.0^{\circ}$, PC2s: $\alpha=30.9^{\circ}$, PC3s: $\left.\alpha=56.7^{\circ} ; P<0.001\right)$, but for FA there was resemblance between the shape change for males PC1 and females PC2 $\left(\alpha=33.9^{\circ}, P<0.0001\right)$ and for corresponding PC3 of both sexes $\left(\alpha=55.1^{\circ}\right.$; $P<0.001)$.

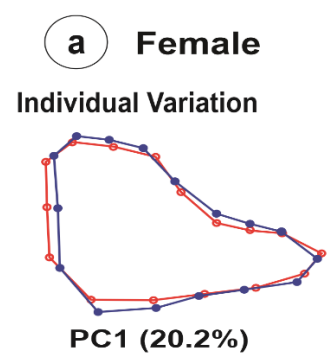

Fluctuating asymmetry

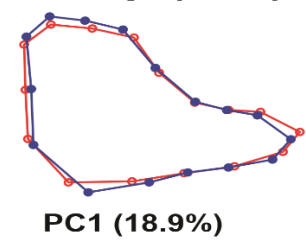

(b) Male

Individual Variation

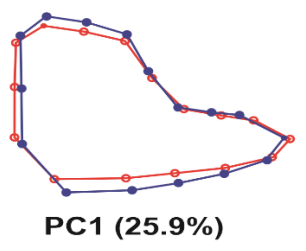

Fluctuating asymmetry

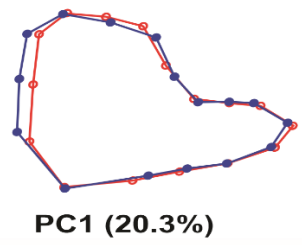

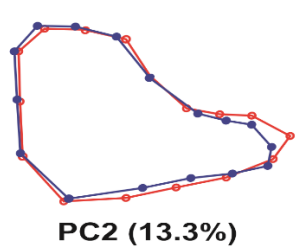

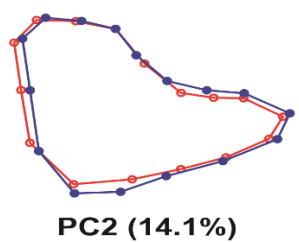

PC2 (14.1\%)

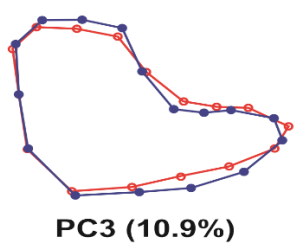

PC3 (10.9\%)

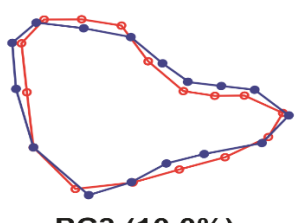

PC3 (10.0\%)

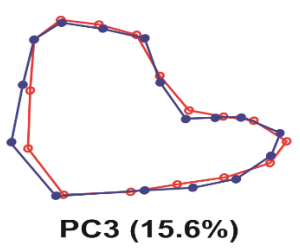

PC2 (17.5\%)

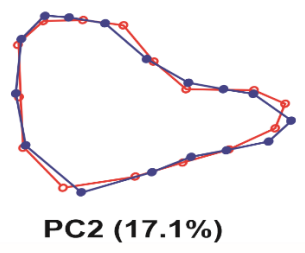

Figure 5: The pattern of individual shape variation and fluctuating asymmetry in chela of C. signatus relative to the first three principal component (PC) scores, after removing the effect of allometry, for female (a) and male (b). The drawings illustrate landmark shifts (blue circles and line) from the average shape (red circles and line) of chelae configurations. The percentage below each diagram shows how much each PC score accounts for the shape variation, however, percentages are not directly comparable because principal component analysis (PCA) performed separately for each of females and males. Wireframe diagrams show the shape for a PC score of 0.1 procrustes unit. 


\section{Integration and modularity}

Manus and pollex for both females and males showed structural integration as indicated by the high and positive PLS pairwise correlation between the two parts. The highest significant PLS correlation showed by PLS1 for individual variation and FA ( $\mathrm{r}_{\mathrm{PLS} 1}$ up to $0.83, P<0.05$ ) followed by PLS2 and PLS3, respectively (Figure 6a,b). However, in both sexes, the $R V$ coefficient was low $(R V \leq 0.3)$ for both individual variation and FA reflecting moderate association between the two parts. For individual variation of both sexes, covariation presented by PLS1 was 50.3\% and $52.8 \%$ of total covariation suggesting that covariation distributed over several axes. Changes for PLS1 of individual variation reflect that when manus shortened, the pollex becomes narrow and slightly extended forwardly for both sexes. For FA of both sexes, data integration was less evident as reflected by low percentage of variation (49\% and $45.4 \%)$ and changes for PLS1. FA reflect that most of covariation found at manus-pollex junction due to change in angulation between two parts (Figure 6a,b).

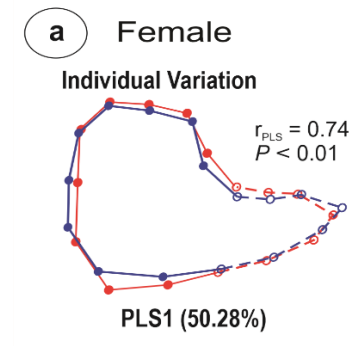

Fluctuating asymmetry
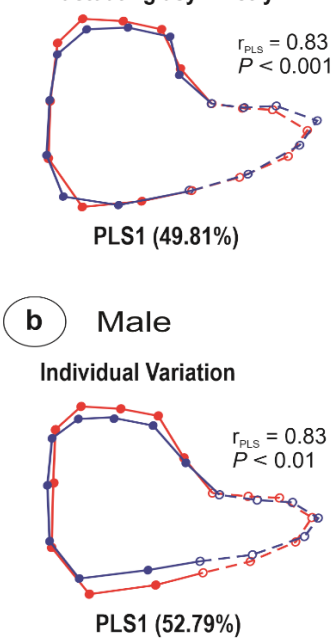

Fluctuating asymmetry

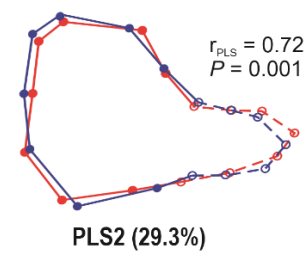

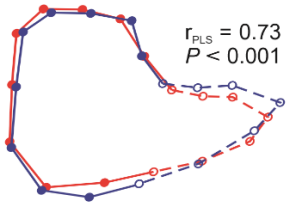

PLS2 (26.56\%)

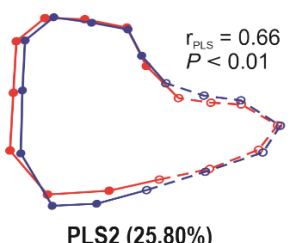

PLS2 (25.80\%)

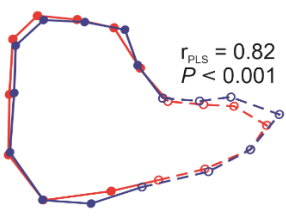

PLS2 (26.15\%)

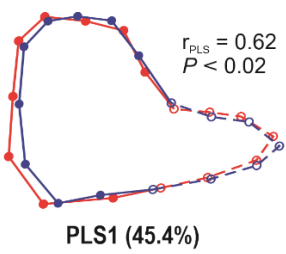

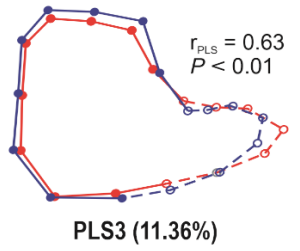

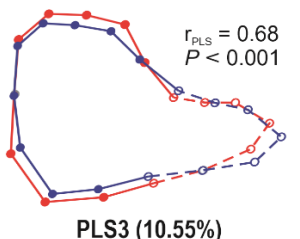

PLS3 (10.55\%)

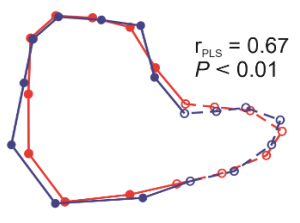

PLS3 (14.01\%)

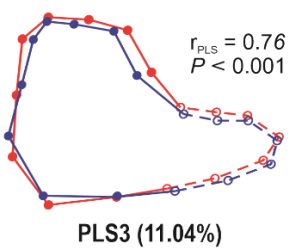

Figure 6: Morphological integration pattern between the manus (solid circles and lines) and pollex (open circles and dash lines) for individual variation and fluctuating asymmetry displayed by pairs of partial least squares (PLS) axes for C. signatus females (a) and males (b). The analysis depends on the residuals from the regression of shape on logtransformed centroid size. Wireframe diagrams show the shape for a PLS score of 0.1 unit. Percentages of the shape variation displayed below each diagram. The drawings illustrate landmark shifts (blue color) from the average shape (red color) of chelae configurations. 
The covariation patterns displayed by PLS and PCA analyses exhibited some accordance, however, there were little differences related to direction and magnitude of landmark motion (Figures 5a,b and $6 a, b)$. For both sexes, angles between the first three pairs of PLS and their corresponding PCA axes for individual variation were small reflecting similarity in pattern of shape variation (females: $\alpha=17.8^{\circ}, 15.5^{\circ}, 38.3^{\circ}, P<0.00001$; males: $\left.\alpha=15.7^{\circ}, 14.7^{\circ}, 25.4^{\circ}, P<0.00001\right)$. Also, for fluctuating asymmetry, the angles between PLS and PCA axes indicate accordance in pattern of shape variation for females $\left(\alpha=16.2^{\circ}, 26.4^{\circ}, 52.8^{\circ}\right.$; $P<0.001)$, but for males there is some differences, where the pair of PLS1 displayed shape change resembles that for PC2, the pair of PLS2 displayed shape changes like those for PC1 and shape changes presented by PLS3 was equivalent to those offered by $\mathrm{PC} 3$ $\left(\alpha:\right.$ PLS1-PC2 $=21.9^{\circ}$, PLS2-PC1 $=31.9^{\circ}$, PLS3-PC3 $\left.=28.9^{\circ} ; P<0.00001\right)$.

Level of shape canalization was somewhat larger for males compared with females (Figure 7), however Levene's test showed no significant differences between sexes $\left(\mathrm{F}_{1,294}=0.22, P=0.64\right)$. As for level of DS, the level of FA was somewhat higher in females than males (Figure 7), and also Levene's test illustrate no significant differences between sexes $\left(\mathrm{F}_{1,294}=0.05\right.$, $P=0.45)$.

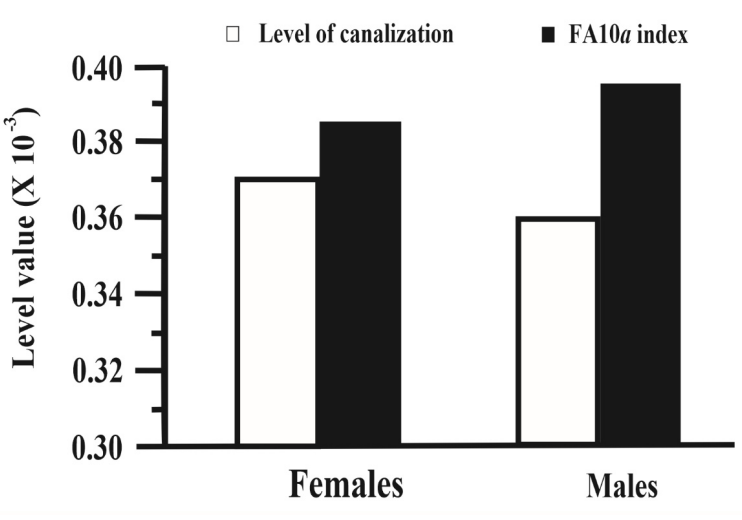

Figure 7: The level of canalization and developmental stability in chelae between the females and males of the hermit crab "C. signatus". The level of canalization was calculated using among individual shape variance, while the level of developmental stability expressed as the level of fluctuating asymmetry shape that was estimated as fluctuating asymmetry index (FA10 $\alpha$ ). Both levels were insignificant between females and males.

\section{Developmental pathway}

The correlations between "individual variation $\times$ FA" covariance matrices (diagonal elements included) for both females and males showed high and significant values (Table 3 ). Therefore, both individual variation and FA shared the same pattern of shape variation. Furthermore, the covariance matrices correlations between females and males regarding individual variation and FA showed significant results suggesting that the pattern of variation was similar in both sexes. Even when diagonal

Table 3: Correlations of covariance matrices and significance of matrix permutation tests between the individual variation (Ind) and fluctuating asymmetry (FA) for right-left chelae of C. signatus.

\begin{tabular}{llll}
\hline & \multicolumn{2}{c}{ Correlation } & \multirow{2}{*}{$P$} \\
\cline { 2 - 3 } & DEI & DEI & \\
\cline { 2 - 3 } Ind $\times$ FA for Females & 0.93 & 0.87 & $<0.0001$ \\
Ind $\times$ FA for Males & 0.87 & 0.62 & $<0.0001$ \\
Ind $\times$ Ind between Females and Males & 0.87 & 0.81 & $<0.0001$ \\
FA $\times$ FA between Females and Males & 0.85 & 0.74 & $<0.0001$ \\
\hline
\end{tabular}

DEI: diagonal elements included; DEE: diagonal elements excluded. 
elements were excluded, the correlations were still somewhat high and significant.

The modularity analysis was executed to judge whether the manus and pollex are separate modules (Figure 8a). Of 11440 considered number of alternative partitions, only 8 and 84 partitions resulted in lower values of $R V$ coefficient for females' individual variation $(R V=0.29, P<0.001)$ and FA $(R V=0.32, P<0.01)$, where these values located on the left side of the distribution (Figure 8b,c). Regarding the males, 239 and 89 partitions of 11440 considered number of alternative partitions showed low values for both individual variation $(R V=0.49, P=0.02)$ and $\mathrm{FA}$ $(R V=0.32, P<0.01)$, which also located on the left side of the distribution graph (Figure 8d,e). Therefore, in both females and males manus and pollex considered as two separate functional modules.

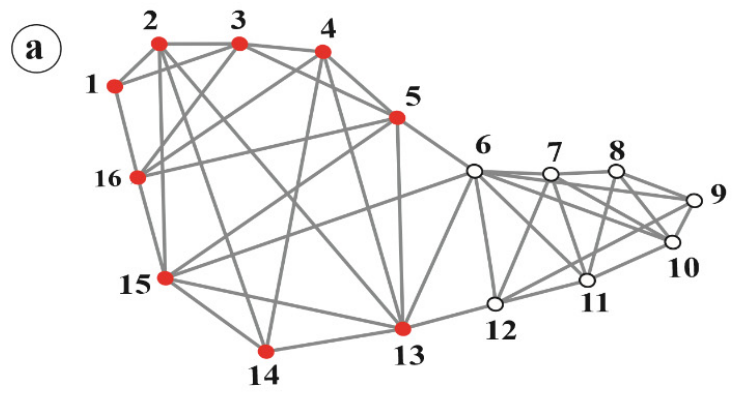

(b) Females
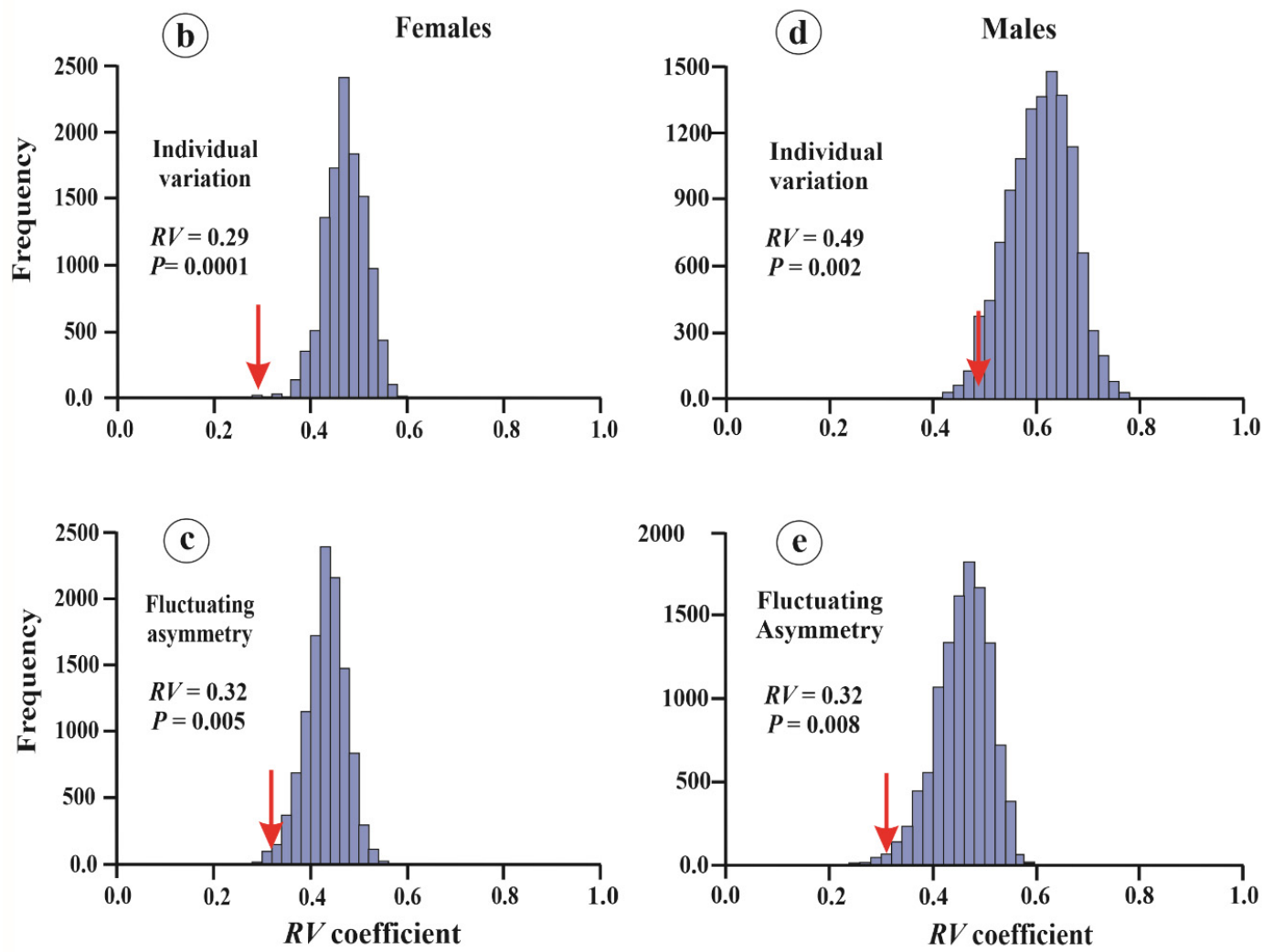

Figure 8: Functional modularity analysis in chela of $C$. signatus after removing the effect of allometry. (a) The tested hypothesis of chela partitioning into two contiguous modules representing the manus (red circle landmarks 1, 2, 3, 4, 5, 13, 14, 15, 16) and the pollex (open circle landmarks $6,7,8,9,10,11,12$ ) parts. Histograms show all possible partitions between two modules for individual variation and fluctuating asymmetry in both females (b and $\mathbf{c}$ ) and males (d and e). The arrows at the left side of the distribution refer to values of Escoufier's $R V$ coefficient $(R V)$ (and corresponding $P$-value) between the two modules. 


\section{DISCUSSION}

The present results confirmed that the chelae shape of hermit crab C. signatus showed the presence of two out of the three asymmetric types, namely DA and FA, using a geometric morphometric approach (GM) based on landmarks. Both types of asymmetry contributed to more than $55 \%$ of the total shape variations. Besides, DA in R-L chelae of both sexes of $C$. signatus showed a similar pattern of shape variations that oriented towards the right because all investigated individuals have bigger right chela, also the degree of DA was higher in males than females.

Heterochely trait (size-heterochely, shape heterochely or both) is vastly displayed in hermit crabs and is genetically fixed in Paguridae (all right-handed). On the other hand, Diogenidae is known as lefthanded hermit crabs, although it contains right-handed hermits ${ }^{[1]}$. Species of the diogenidean genus Clibanarius displayed both symmetrical chelae and asymmetrical chelae, which was recorded as righthanded or left-handed (i.e., directional asymmetry $)^{[1,18]}$. The chelae symmetry or asymmetry that was reported for members of genus Clibanarius depend on the size ${ }^{[18]}$, but not the shape and thus may hide a superficial homochelous. Also, reversal of handedness was not reported before in this species, which does not mean its absence particularly that the investigated $C$. signatus specimens were chosen to be healthy (see materials and methods).

Individuals of $C$. signatus showed equal-sized chelae during development, which in adults become subequal showing asymmetry where right chela somewhat is bigger ${ }^{[40,41]}$. This asymmetry in adulthood is an indicative of the transition from symmetry to direct asymmetry ${ }^{[40,41]}$ and thus right-handedness isn't a property of C. signatus at birth. In the absence of genetic information for handedness in C. signatus, it can't be judged that the right-handed is a genetically fixed trait. Other crustaceans showed that asymmetry of chelae begins after settlement of juveniles due to characteristic exercises which produce muscle differentiation ${ }^{[42,43]}$.

Generally, the right and left chelae formed by the same genome, and their symmetry in the post-larval stage reflect the crab's ability to canalize development against pressures ${ }^{[44]}$, but the appearance of chelae asymmetry in adults illustrate that developmental trajectory was changed developmentally or genetically under environmental stress, which breaks the symmetry in a fixed direction ${ }^{[6,10,26]}$. The environmental components, that may break symmetry and lead to heterochely in adults of $C$. signatus, may be the dextral shell architecture, foraging behavior and intra/interspecific competitions ${ }^{[3]}$.

It is widely recognized that severe environmental pressures of the Red Sea intertidal flats, particularly those related to temperature, salinity and presence of predators, might force the individuals of C. signatus to withdraw completely inside the $C$. caerulum shells. Therefore, the chelae of hermit crabs may be affected by shell architecture and receive more stress in the dextral shell. In addition, the individual crab closes the shell narrow aperture with one of its chelae to avoid environmental conditions or predators. This may also explain the right chela enlargement compared with the left one in proportion to narrow shell aperture. According to the results of Abd El-Wakeil et $a .^{[45]}$, the growth of $C$. signatus was affected by shell architecture, particularly right chela which significantly affected by shell aperture length and width, as well as, showed a better proportional fit for protection than do left chela. In this regard, Turra and Leite ${ }^{[46]}$ mentioned that individuals of hermit crab Pagurus criniticornis showed more right-left chelae asymmetry in shells of Cerithium spp. than other tested shells, where the right chela was larger than the left one. Also, hermits in medium-spire shells are subjected more to chelae asymmetry than those in low-spire shells ${ }^{[46]}$.

In contrast, Imafuku and Ikeda ${ }^{[47]}$ suggested that handedness was not linked 
to the architecture of occupied shells based on that chelae are usually maintained outside of the shell, which weakens the stresses they received by the occupied shell, also handedness in hermit crab Pagurus filholi was not related to utilize dextral shell since several individuals were right-handed and others are left-handed.

Competition among hermit crabs over shells and male-male fights for mating can lead to enlargement the chelae, where the larger chela in the heterochely crabs has a greater mechanical advantage and a larger closing muscles volume than the smaller chela ${ }^{[47]}$. Therefore, the presence of DA in the present hermit crab may also be explained as a result of asymmetrical development of chela muscles because of a higher frequency using of the right chela in fighting over suitable and preferred shells. But clustering behavior of the present species suggests that the association between DA and performance of specific activities as mating or competition over shells is somewhat weak where suitable shells are available and easy to obtain by hermits ${ }^{[48]}$. Therefore, the second hypothesis of Pélabon and Hansen ${ }^{[49]}$, where the slight enlargement of a right chela is a correlated response to various forms of indirect selection, and are therefore not adaptations may be better explanation for the present result. Another point of view proposed by Hermisson et al. ${ }^{[50]}$, in which the subtle directional asymmetry can result from stabilizing selection on epistatic genetic, where the genes responsible for increasing directional asymmetry inhibit or diminish the effect of each other. Positive and significant correlation between corresponding DA vectors of landmarks displacements for two sexes suggests a genetic basis that appeared during the developmental process and lead to directional asymmetry ${ }^{[6,26]}$.

Chelae of the present species reflect the presence of sexual dimorphism in both its size and shape. Generally, chelae-size sexual dimorphism is evident in hermit crabs showing variant degrees of sexual dimorphism expressions ${ }^{[51]}$ and reflect favoring of sexual selection to the large size of chela for sexual signaling and fighting for mates suggesting that larger chela is adaptive in males ${ }^{[51]}$.

Among asymmetry types, FA regarded as a developmental perturbation ${ }^{[5,6,10]}$ and thus is useful to reflect the amount of stress that animal subject to. Chelae shape variations explained by FA were higher in both sexes of $C$. signatus than DA. Although FA revealed sex-differences as indicated by percentages in Table "1" and values of FA10 $a$ index, where females showed higher value than males, this sex difference was insignificant. This suggests that two sexes subjected, more or less, to the same level of stress. This was expected due to occupation of the same shell type which denotes the shell architecture as a source for environmental stress compared with other sources for asymmetry. However, the previous results may reveal the existed differences in the levels of developmental homoeostasis between males and females, which in turn reflect the stresses faced by the hermit individuals in both sexes during changing shells ${ }^{[45,47]}$.

It is widely approved that the need of hermit crabs' individuals to change their occupied shells during growth and looking for suitable ones, where inadequate shells cause modifications in the crab body form $^{[46]}$ and affect its growth ${ }^{[47]}$. Therefore, FA detected in chela of both sexes of C. signatus possibly result from a stressful development and consequently high developmental instability related to the alternative pathway. In addition, the higher FA level in females than males may reflect the higher stress they face during intraspecific competition for the better shells that fulfill a large space for egg incubation $^{[17,44]}$.

The present work revealed that the most affected part in chelae of both sexes was manus and this return to the muscle mass which is large and more size-stable in males compared with its fluctuation in females reflecting developmental instability in females. Indeed, if the females occupied 
low-quality shells than males, especially shell with narrower opening, this might affect the muscle size after molting and during growth, and thus females subjected to more stress.

In the present work, PCA presented distinct patterns of covariations among landmarks of individual variation and FA. For females, most of these displayed by PC1 and could attribute to the displacement of landmarks at the dorsal margin of manus (landmarks 1-4), at the ventral margin of manus (landmark 14) and the tip of pollex (landmarks 9-10). As for males, most of the covariations displayed by $\mathrm{PC} 1$ could be attributed to displacement of landmarks at the dorsal margin of manus (landmarks 1, 3-4) and the ventral margin of manus (landmark 14). Furthermore, the similarity between the covariance matrices of fluctuating asymmetry and individual variation, which reflected by covariance correlation and assured by angles between corresponding PCs suggested that the developmental processes in R-L chelae might affect both asymmetries ${ }^{[13,15]}$. However, variability of PCs percentages, which accounted for variations of individual variation and fluctuating asymmetry, revealed an incomplete congruence. This suggested that developmental processes may respond dissimilarly to genetic or environmental variations between individuals than to random differences between the two chelae as proposed by Klingenberg ${ }^{[13]}$.

Finally, developmental instability at individual levels is expected for chela of C. signatus, resulting in the elevation of FA level, besides, the difference in genetic basis between sexes might cause the chelae to be more or less sensitive to the stress produced by occupied shells. Therefore, the quality of occupied shells and the fitness of hermit crab individuals might put the frame for the relationship between FA and chelae shape $^{[27]}$. Studying FA of natural populations is problematic because many environmental stressors have effects on individuals in a natural population, that still need to be tested as salinity, temperature, predators, exposure to air during low tide and others.

The present results reflect a significant and moderate shape integration between manus and pollex of chela as indicated by the $R V$ coefficient (i.e., chela is an integrated anatomical structure). The analysis of morphological integration in chela of $C$. signatus showed similar covariation patterns for FA and individual variations as indicated by high PLS correlations.

Also, PC scores for both FA and individual variation reflect similar patterns of variations in manus and pollex with minor differences in between, therefore the same patterns account for different amounts of variation at the two levels of morphological variation $^{[8]}$. This means that FA transmitted directly through developmental pathways between the two parts ${ }^{[12]}$ due to that the manus and pollex share the effects of the same disturbance. Generally, phenotypic integration of the two chela parts means that selection on a particular size and shape of one part could result in correlated responses in the other part ${ }^{[16]}$. Also, the high correlations between manus and pollex reflect the power of the associations between sets of landmarks in two parts and illustrate that covariation is found across the entire chela for individual variation and FA. Integration of FA is usually related to adjacent structures because the interaction of these structures facilitates transmitting of disturbance between them ${ }^{[12]}$.

The similarity in FA $\times$ individual variation patterns refer to the developmental integration of neighboring compartments of chela, i.e., manus and pollex. This implies that direct interactions of developmental pathways might be the controlling mechanism between developmental pathways and the main process that also causes trait covariation among individuals ${ }^{[33]}$.

The integration of shape variation was clear anywhere of the chela for females as illustrated by correspondence between the PCs of chela variation and PLS axes of covariation between manus and pollex, 
which was identical, as well as from the comparison of angles between PCs and PLS axes. However, males showed somewhat similarity due to not identical correspondence between PCs and PLS axes. The correspondence between PCs and PLS axes revealed that integrated features are among the dominant features of chela shape variation and covariation between two parts of chela explain a large part of the whole chela variation $^{[38]}$. Therefore, morphological integration illustrated by the high correlation between manus and pollex revealed that these two parts don't grow independently of each other and subtle random disturbances are transmitted across the developing chela $^{[8]}$. FA integration between two chela parts requires developmental interactions to transmit the effects of the disturbance to both parts. Possible explanation for these interactions include the embryonic origin of parts $^{[13]}$ or differential use of right and left chelae $^{[15,16]}$.

The integrated traits reflect the degrees of phenotypic stability and thus might reflect responses to environmental stress. This reveals the relationship between morphological integration and both canalization and developmental stability ${ }^{[52]}$. The present species showed moderate morphological integration in the chela, which may mean that environmental stress on the chela was moderate. The subtle asymmetry was probably due to less stress exposed by occupied shells in a way that influence chela development. In the same context, the availability and suitability of shells from the time of occupation by hermits after the post-larval stage and throughout their development could potentially reduce stress exposure for hermits $^{[45,48]}$.

The correspondence between covariance matrices of "individual variation $\times F A$ " for both sexes reflects that the same developmental processes are responsible for canalization and developmental stability ${ }^{[33]}$. Studies on the similarity between the patterns of covariation for FA and individual variation concluded that FA reflects canalization $^{[52]}$. In the present work, both sexes of $C$. signatus showed more or less same level of canalization for chelae shape variance, which suggests that they possess the same genetic and environmental component of variance that may result in equality in the variability and therefore minimize the phenotypic variance between their chelae ${ }^{[50,52]}$.

Testing Manus-pollex integration for functional modularity revealed that these structures are two modules reflecting differences in functional specializations between them. Manus is larger and supported with closer and opener muscles, which control movements of dactyl. Pollex is conical and supported with small teeth on its inner margin and functionally help dactyl in gathering food materials ${ }^{[53]}$. Therefore, manus and pollex modules of $C$. signatus chelae represent different functions performed by the two parts which may be more related to the feeding mechanism. During feeding, the muscles in the manus part load the power to open and close the dactyl that, with the help of pollex, grasp and carry food items toward the mouth $^{[53]}$. Therefore, the functionality of manus-pollex depends on the correlation between them, which was illustrated by PLS analysis. The previous analysis showed that manus-pollex integration explained up to $80 \%$ of the shape variation. Consequently, it can be proposed that the morphology of manus and pollex will vary forming functional units that are important in applying capability and pressure during grasping and carrying food items.

Integration with and without modularity was reported for some structures in both vertebrates and invertebrates ${ }^{[11,15,16,32,35]}$. Generally, it is difficult to compare results among these studies owing to various morphometric methods and used principles of integration and modularity.

\section{FUNDING SOURCE DISCLOSURE}

This research received no specific grant from any funding agency in the public, commercial, or not-for-profit sectors. 


\section{CONFLICT OF INTEREST}

The author declares no potential financial conflict of interest.

\section{ACKNOWLEDGMENTS}

The author thanks Prof. Dr. Azza M. Khdre (Zoology Department, Faculty of Science, Sohag University) for her helpful advices during writing this Paper.

\section{REFERENCES}

[1] McLaughlin, P. A. (2003). Illustrated keys to families and genera of the superfamily Paguroidea (Crustacea: Decapoda: Anomura), with diagnoses of genera of Paguridae. Mem Mus Vic, 60(1): 111-144.

[2] Billock, W. L. and Dunbar, S. G. (2009). Influence of motivation on behaviour in the hermit crab, Pagurus samuelis. J Mar Biol Assoc UK, 89(4): 775-779.

[3] Yasuda, C.; Suzuki, Y. and Wada, S. (2011). Function of the major cheliped in male-male competition in the hermit crab Pagurus nigrofascia. Mar Biol, 158: 2327-2334.

[4] Marques, J. F.; Costa, J. L. and Cabral, H. N. (2005). Variation in bilateral asymmetry of the Lusitanian toadfish along the Portuguese coast. J Appl Ichthyol, 21(3): 205-209.

[5] Palmer, A. R. and Strobeck, C. (1992). Fluctuating asymmetry as a measure of developmental stability: implications of non-normal distributions and power of statistical tests. Acta Zool Fenn, 191: 57-72.

[6] Palmer, A. R. and Strobeck, C. (2003). Fluctuating asymmetry analyses revisited. In: Developmental Instability (DI): Causes and Consequences (Polak, M. ed), pp. 279319. Oxford University Press, Oxford, UK.

[7] Dongen, S. V. (2006). Fluctuating asymmetry and developmental instability in evolutionary biology: past, present and future. J Evol Biol, 19: 1727-1743.
[8] Klingenberg, C. P. and Nijhout, $\mathrm{H}$. F. (1999). Genetics of fluctuating asymmetry: a developmental model of developmental instability. Evolution, 53: 358-375.

[9] Eeva, T.; Tanhuanpää, S.; Råbergh, C. et al. (2000). Biomarkers and fluctuating asymmetry as indicators of pollution-induced stress in two holenesting passerines. Funct Ecol, 14(2): 235-243.

[10] Ivanković Tatalović, L.; Anđelić, B.; Jelić, M. et al. (2020). Fluctuating asymmetry as a method of assessing environmental stress in two predatory carabid species within mediterranean agroecosystems. Symmetry, 12(11): 1890-1907.

[11] Klingenberg, C. P. (2009). Morphometric integration and modularity in configurations of landmarks: tools for evaluating a priori hypotheses. Evol Dev, 11: 405-421.

[12] Klingenberg, C. P. (2008). Morphological integration and developmental modularity. Annu Rev Ecol Evol Syst, 39: 115-132.

[13] Klingenberg, C. P. (2003). Developmental instability as a research tool: using patterns of fluctuating asymmetry to infer the developmental origins of morphological integration. In: Developmental Instability: Causes and Consequences (Michal, P. ed), pp. 427-442. Oxford University Press, Oxford, UK.

[14 Klingenberg, C. P. (2005). Developmental constraints, modules, and evolvability. In: Variation (Hallgrímsson, B. and Hall, B. eds), pp. 219-247. Elsevier, Amsterdam, Netherlands.

[15] Klingenberg, C. P. and Zaklan, S. D. (2000). Morphological integration between developmental compartments in the Drosophilawing. Evolution, 54: 1273-1285.

[16] Klingenberg, C. P.; Mebus, K.; Auffray, J.-C. (2003). Developmental integration in a complex morpho- 
logical structure: How distinct are the modules in the mouse mandible? Evol Dev, 5: 522-531.

[17] Dongen, S. V. (2006). Fluctuating asymmetry and developmental instability in evolutionary biology: past, present and future. J Evol Biol, 19: 1727-1743.

[18] Rahayu, D. L. (2003). Hermit crab species of the genus Clibanarius (Crustacea: Decapoda: Diogenidae) from mangrove habitats in papua, Indonesia, with description of a new species. Mem Mus Vic, 60: 99-104.

[19] Rohlf, F. J. (2010). TpsDig2: Digitize Coordinates of Landmarks and Capture Outlines. Department of Ecology \& Evolution, Stony Brook Univerity, Stony Brook, NY, USA.

[20] Hopkins, M. J. and Thurman, C. L. (2010). The geographic structure of morphological variation in eight species of fiddler crabs (Ocypodidae: Genus $U c a$ ) from the eastern United States and Mexico. Biol J Linn Soc, 100: 248-270.

[21] Zelditch, M. L.; Swiderski, D. L. and Sheets, H. D. (2012). Geometric morphometrics for biologists: A primer. Academic press, Cambridge, MA, USA.

[22] Klingenberg, C. P.; Barluenga, M. and Meyer, A. (2002). Shape analysis of symmetric structures: quantifying variation among individuals and asymmetry. Evolution, 56: 1909-1920.

[23] Graham, J. H.; Raz, S.; Hel-Or, H. et al. (2010). Fluctuating asymmetry: methods, theory, and applications. Symmetry, 2: 466-540.

[24] Marques, M. (2007). SAGE: Symmetry and Asymmetry in Geometric Data Version 1.21. University of Michigan, Ann Harbor, MI, USA.

[25] Klingenberg, C. P. (2011). MorphoJ: an integrated software package for geometric morphometrics. Mol Ecol Resour, 11: 353-357.
[26] Palmer, A. R. (2012). Developmental origins of normal and anomalous random right-left asymmetry: lateral inhibition versus developmental error in a threshold trait. Contrib Zool, 81: 111-124.

[27] Klingenberg, C. P. (2015). Analyzing fluctuating asymmetry with geometric morphometrics: concepts, methods, and applications. Symmetry, 7: 843934.

[28] Good, P. (2013). Permutation tests: a practical guide to resampling methods for testing hypotheses. Springer Science \& Business Media, Berlin, Germany.

[29] Klingenberg, C. P. (2014). Size, shape, and form: concepts of allometry in geometric morphometrics. Dev Genes Evol, 226: 113-137.

[30] Cardini, A. (2019). Integration and modularity in procrustes shape data: is there a risk of spurious results? Evol Biol, 46(1): 90-105.

[31] Klingenberg, C. P.; McIntyre, G. S. and Zaklan, S. D. (1998). Left-right asymmetry of fly wings and the evolution of body axes. Proc R Soc Lond B Biol Sci, 265: 1255-1259.

[32] Klingenberg, C. P. and MarugánLobón, J. (2013). Evolutionary covariation in geometric morphometric data: analyzing integration, modularity, and allometry in a phylogenetic context. Syst Biol, 62: 591-610.

[33] Jojić, V.; Blagojević, J. and Vujošević, M. B. (2011). Chromosomes and cranial variability in yellow-necked field mice (Apodemus flavicollis). $\mathrm{J}$ Mammal, 92: 396-406.

[34] Rohlf, F. J. and Corti, M. (2000). Use of two-block partial least-squares to study covariation in shape. Syst Biol, 49: 740-753.

[35] Adams, D. C. and Collyer, M. L. (2016). On the comparison of the strength of morphological integration across morphometric datasets. Evolution, 70: 2623-2631. 
[36] Ren, J.; Bai, M.; Yang, X. K. et al.(2017). Geometric morphometrics analysis of the hind wing of leaf beetles: proximal and distal parts are separate modules. ZooKeys, 685:131149.

[37] Urošević, A.; Ljubisavljević, K. and Ivanović, A. (2015). Fluctuating asymmetry and individual variation in the skull shape of the common wall lizard (Podarcis muralis Laurenti, 1768) estimated by geometric morphometrics. Herpetol J, 25: 177-186.

[38] Klingenberg, C. P. and MarugánLobón, J. (2013). Evolutionary covariation in geometric morphometric data: analyzing integration, modularity, and allometry in a phylogenetic context. Syst Biol, 62: 591-610.

[39] Willmore, K. E.; Leamy, L. and Hallgrímsson, B. (2006). Effects of developmental and functional interactions on mouse cranial variability through late ontogeny. Evol Dev, 8: 550-567.

[40] Tirmizi, N. M. and Siddiqui, F. A. (1979). The larval development of Clibanarius signatus Heller and C. virescens (Krauss) (Decapoda: Diogenidae) under laboratory conditions. Pak J Zool, 11: 239-261.

[41] Bartilotti, C.; Calado, R. and Dos Santos, A. (2008). Complete larval development of the hermit crabs Clibanarius aequabilis and Clibanarius erythropus (Decapoda: Anomura: Diogenidae), under laboratory conditions, with a revision of the larval features of genus Clibanarius. Helgol Mar Res, 62: 103121.

[42] Longo, M. V.; Goldemberg, A. L. and Díaz, A. O. (2011). The claw closer muscle of Neohelice granulata (Grapsoidea, Varunidae): a morphological and histochemical study. Acta Zool, 92(2): 126-133.

[43] Goldstein, J. S. and Tlusty, M. F. (2003). Substrate determinants and developmental rate of claw asymmetry in American lobsters, Homarus americanus. J Crust Biol, 23(4): 890896.

[44] Benítez, H. A.; Lemic, D.; VillalobosLeiva, A. et al. (2020). Breaking symmetry: fluctuating asymmetry and geometric morphometrics as tools for evaluating developmental instability under diverse agroecosystems. Symmetry, 12(11):17891801.

[45] Abd El-Wakeil, K. F.; Said, E. A.; Obuid-Allah, A. H. et al. (2010). Gastropod shell occupation by hermit crab Clibanarius signatus (Crustacea: Decapoda) on the Red Sea Coast, Egypt. Assiut Univ J Zool, 30: $1-10$.

[46] Turra, A. and Leite, F.P. (2003). The molding hypothesis: linking shell use with hermit crab growth, morphology, and shell-species selection. Mar Ecol Prog Ser, 265: 155-163.

[47] Imafuku, M. and Ikeda, H. (2014). Asymmetrical morphology and growth of the hermit crab Pagurus filholi (Decapoda, Anomura, Paguridae) reared in non-dextral shell conditions. Crustaceana, 87: 476-488.

[48] Ismail, T. G. (2011). Behavioural activities of five hermit crabs from Egyptian Red Sea coast. J Egypt Ger Soc Zool, 63:153-179.

[49] Pélabon, C. and Hansen, T. F. (2008). On the adaptive accuracy of directional asymmetry in insect wing size. Evol Int J Org Evol, 62: 28552867.

[50] Hermisson, J.; Hansen, T. F. and Wagner, G. P. (2003). Epistasis in polygenic traits and the evolution of genetic architecture under stabilizing selection. Am Nat, 161: 708-734.

[51] Fransozo, A.; Garcia, R. B. and Mantelatto, F. L. M. (2003). Morphometry and sexual maturity of the tropical hermit crab Calcinus tibicen (Crustacea, Anomura) from Brazil. J Nat Hist, 37(3): 297-304. 
[52] Hallgrímsson, B.; Willmore, K. and Hall, B. K. (2002). Canalization, developmental stability, and morphological integration in primate limbs. Am J Phys Anthropol, 119: 131-158.
[53] Schembri, P. J. (1982). Feeding behaviour of fifteen species of hermit crabs (Crustacea: Decapoda: Anomura) from the Otago region, southeastern New Zealand. J Nat Hist, 16: 859-878.

\section{How to cite this article:}

Ismail, T. G. (2021). A geometric-morphometric analysis of the heterochely in the Red Sea hermit crab "Clibanarius signatus", and its developmental stability and modularity. Egyptian Journal of Zoology, 76: 30-51 (DOI: 10.21608/ejz.2021.77508.1056). 


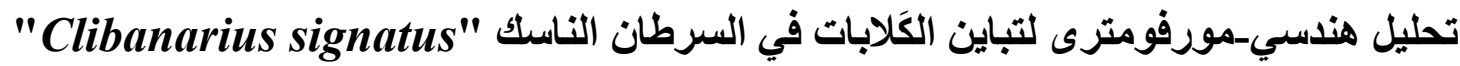
بالبحر الأحمر واستثرار نمائها ونمطيتها

\author{
طارق جادالكريم إسماعيل \\ قسم علم الحيوان، كلية العلوم، جامعة سوهاج، سوهاج، جمهورية مصر العربية
}

يستخدم التحليل الهندسي-المورفومتري بشكل متز ايد لتحديد وتحليل الأشكال البيولوجية والتمييز بين الاختلافات الثكلية

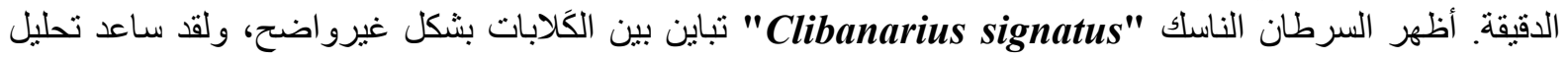

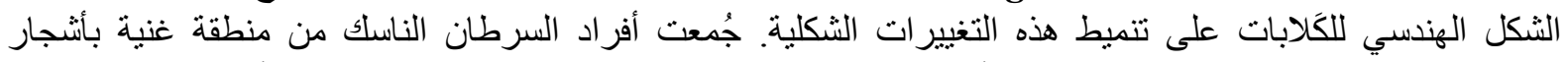

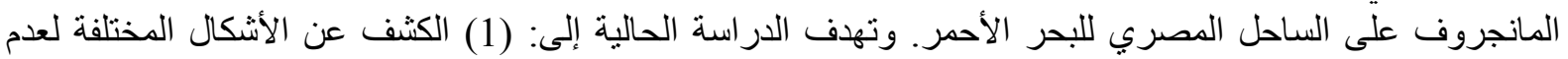

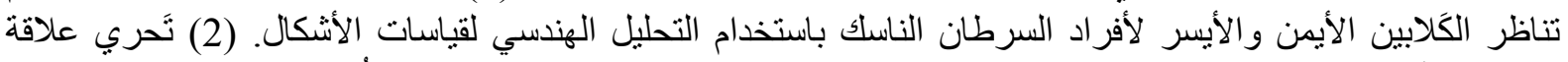

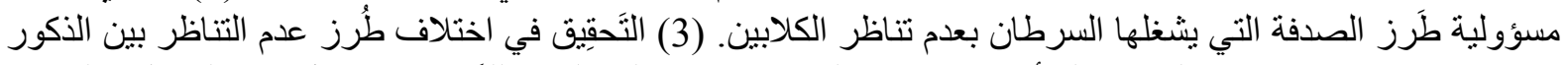

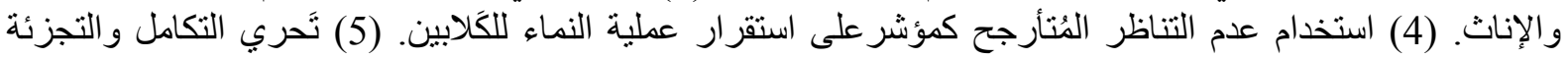

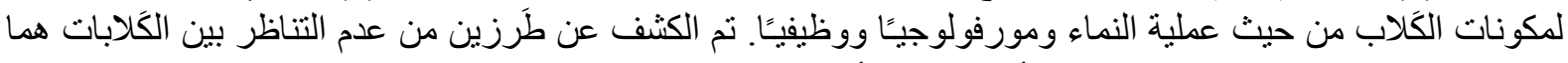

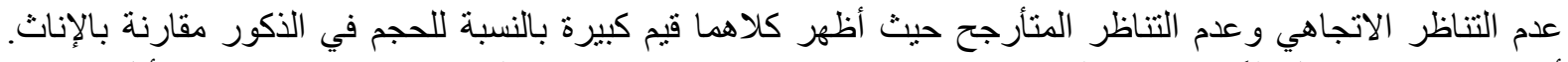

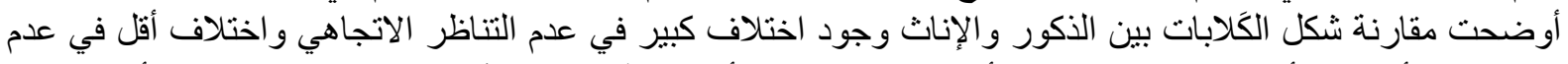

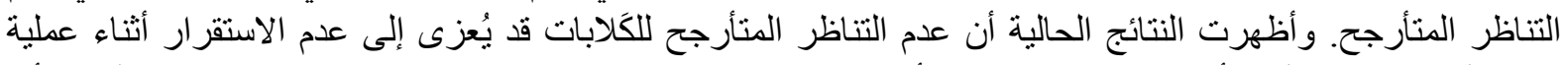

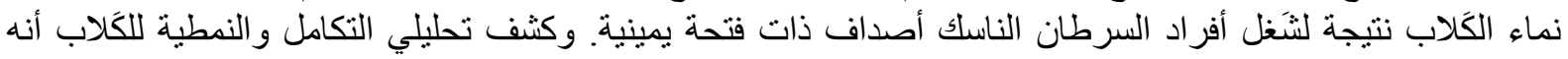

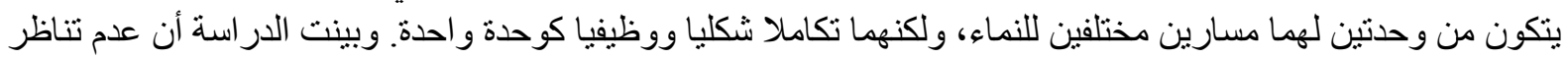

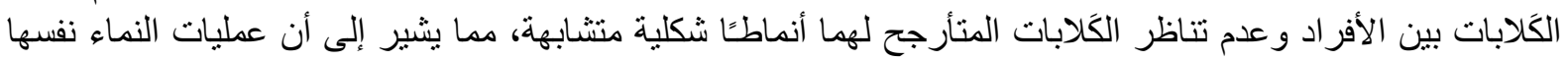

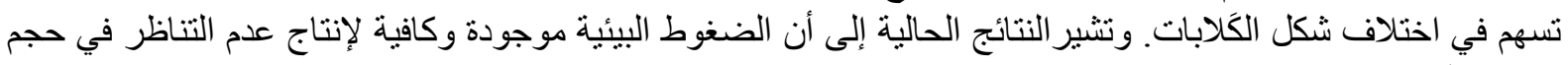
وشكل الكَلابات. 